\title{
All hands on DEK
}

\author{
David M. Bodine
}

Genetics and Molecular Biology Branch, National Human Genome Research Institute, Division of Intramural Research, NIH, Bethesda, Maryland, USA

\begin{abstract}
In this issue of the $\mathrm{JCl}$, Capitano et al. demonstrate that the secreted form of the DNA-binding chromatin factor DEK regulates hematopoiesis. It is known that DEK can be secreted by macrophages and other cells, but no function has been attached to secreted DEK. Capitano et al. showed that extracellular DEK activates signaling through the CXCL2 receptor, which in turn enhances the proliferation of hematopoietic stem cells and decreases hematopoietic progenitor cell numbers both in vivo and in vitro. These results offer the opportunity to expand transplantable stem cells to improve outcomes in patients undergoing bone marrow transplant.
\end{abstract}

\section{DEK-mediated regulation of hematopoiesis}

In this issue of the JCI, Capitano et al. present a fascinating study showing that secreted DEK, an endogenous DNA-binding chromatin factor, regulates hematopoiesis through CXCR2 chemikone signaling, both in vitro and in vivo (1). At first glance, $D E K$ would not seem to be a candidate to be such a regulator. DEK was originally described as a fusion partner with the nuclear pore complex (NUP214) gene, forming the DEK-NUP214 fusion gene in a subset of acute myeloid leukemia (AML), and it has been classified as an oncogene (2-4). DEK has been shown to be a DNA-modulating protein involved in global heterochromatin integrity, DNA replication, DNA repair, RNA transcription, and RNA splicing. DEK is highly expressed in cells from most human tissues, especially hematopoietic cells, and overexpressed in many tumors $(5,6)$.

Recently it has been shown that DEK can be secreted by macrophages, particularly in response to IL-8, where it serves to attract neutrophils, CD8 ${ }^{+} \mathrm{T}$ lymphocytes, and natural killer cells, among other functions (7-9). Consistent with a proinflammatory function, DEK is also found in synovial fluid samples from patients with juvenile arthritis (10). In this regard, DEK is similar to another DNA-modulating protein, HMGB1, which after reaching the extracellular space, triggers inflammation and the recruitment of macrophages to a tissue-healing phenotype, as well as the proliferation of hematopoietic stem cells (HSCs) and hematopoietic progenitor cells (HPCs) (11). In their article, Capitano et al. show that secreted DEK regulates the proliferation of HSCs and HPCs in vitro and in vivo. Unlike secreted HMGB1, which appears to exert its effects strictly through chromatin modulation, DEK alters the proliferation of HSCs and HPCs as a hematopoietic cytokine.

\section{Mechanism of DEK action in vivo and in vitro}

Capitano et al. show that extracellular DEK enhances the expansion of human and mouse HSCs and decreases HPC numbers in vivo and in vitro. They demonstrate that DEK exerts these functions through the CXCR2 receptor. Mutant forms of DEK lacking the nuclear localization signal or DNA-binding domain also altered HSC and HPC numbers in vivo or in vitro. The authors conclude that DEK acts as a hematopoietic cytokine, one that has abilities that are clearly clinically relevant. The

\section{Delated Article: p. 2555}

Conflict of interest: The author has declared that no conflict of interest exists.

DEK-induced proliferation of HSCs offers an opportunity to expand transplantable stem cells to improve transplant outcomes in patients, one of the holy grails of stem cell biology. These findings are summarized in Figure 1.

Three aspects distinguish the studies by Capitano et al.: their innovation, the depth of the mechanistic experiments, and the potential for clinical application. These are the first studies to our knowledge to define a fundamental function for DEK in normal hematopoiesis. The nuclear regulatory functions of intracellular DEK are relatively well understood in tumor cells, and the secretion of DEK by different hematopoietic cells has been described in the context of inflammation. However, the function of secreted DEK in normal hematopoiesis has not been explored. Capitano et al. show that secreted DEK plays a critical role in the regulation of normal hematopoiesis. Their findings have introduced a paradigm for stem cell regulation with the potential to influence the field for years to come.

Mechanistically, the study is robust and compelling. The authors functionally demonstrate that extracellular DEK expands human and mouse HSCs using the conventional SCID-repopulating cell (SRC) and mouse competitive repopulation assays, regarded as the gold standard in the field. Beyond this evidence, Capita-

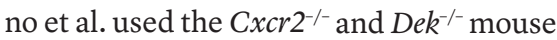
models to rigorously demonstrate that secreted DEK, rather than intracellular or nuclear DEK, is responsible for the alteration in HSC and HPC proliferation. Furthermore, the authors show that inhibitors of CXCR2, and/or the coreceptor heparan sulfate proteoglycan, can block these functions in vivo and in vitro. The authors demonstrate that the activation of the phosphorylation pathways (phosphorylated ERK1/2, AKT, and p38 MAPK) by CXCR2 signaling is required to achieve the effects on stem and progenitor cell numbers. In additional experiments, mutant DEK proteins lacking the DNA-binding domain or the nuclear localization signal 
Effects of extracellular DEK on hematopoiesis

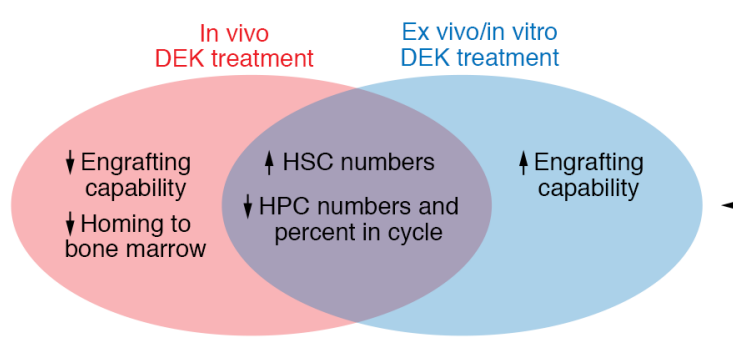

Proposed mechanism

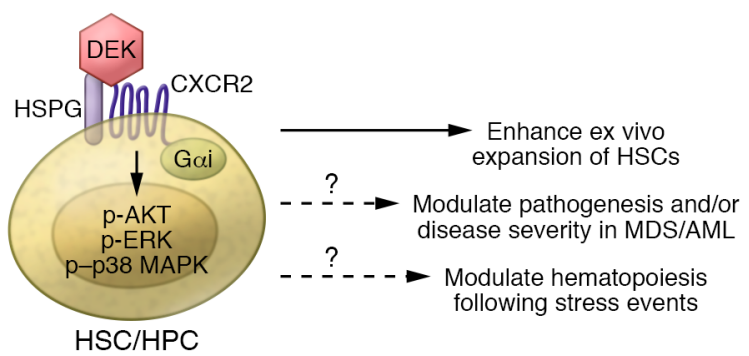

Figure 1. The role of extracellular DEK in hematopoiesis. Extracellular DEK engages the CXCR2 receptor and heparan sulfate proteoglycans (HSPG), triggering a signaling cascade that phosphorylates AKT, ERK, and p38 MAPK (center). DEK increases hematopoietic stem cell (HSC) and decreases hematopoietic progenitor cell (HPC) proliferation in vivo and in vitro, with conflicting effects on HSC engraftment (left). Potential clinical applications are shown on the right.

were as effective as WT DEK in eliciting the expansion of HSCs in vitro.

Notably, Capitano et al. went on to show that nuclear reorganization, which also occurs in cells treated with WT DEK, is not responsible for the effects on HSC and HPC proliferation. The authors document that treatment with extracellular DEK leads to changes in chromatin, which they support by demonstrating changes in the transcriptional profile and biochemical analyses that show the activation of critical regulators of hematopoiesis. Importantly, mutant versions of the DEK protein that do not enter the nucleus or bind DNA still exert their effects on hematopoiesis, excluding nuclear reorganization as a mechanism for the proliferation effects.

\section{Doors opened for future research}

Finally, Capitano discuss the clinical and research opportunities that may arise from their work. DEK treatment increases the number of HSCs, both phenotypically and functionally, by allowing HSC proliferation while inhibiting differentiation. While many operational hurdles remain, the ability to expand stem cells without simultaneously losing large portions of them to differentiation is a paradigm-shifting application. For example, because the number of HSCs in a single umbilical cord blood (UCB) unit is usually limited, the expansion of HSCs from UCB would mean that a single donor could be used, greatly simplifying both donor selection and graft management. These findings could also lead to a better understanding of the pathogenesis of myelodysplastic syndrome (MDS) and AML. The authors also discuss the loss of engraftment of HSCs from DEK-treated mice in spite of the increase in functional HSCs. They speculate that DEK may be having effects on other cells in the niche (expressing CXCR4?) and discuss how this could be used clinically. There is the potential for a career's worth of new experiments to arise from this work, and "all hands will be on DEK" to extend these observations.

Address correspondence to: David $\mathrm{M}$. Bodine, 49 Convent Drive, MSC 4442, Bethesda, Maryland 20892-4442, USA. Phone: 301.402.0902; Email: tedyaz@ mail.nih.gov.

1. Capitano ML, et al. Secreted nuclear protein DEK regulates hematopoiesis through CXCR2 signaling. J Clin Invest. 2019;129(6):2555-2570.

2. Soekarman D, et al. Dek-can rearrangement in translocation (6;9)(p23;q34). Leukemia. 1992;6(6):489-494.

3. Soekarman D, et al. The translocation $(6 ; 9)$ (p23;q34) shows consistent rearrangement of two genes and defines a myeloproliferative disorder with specific clinical features. Blood. 1992;79(11):2990-2997.

4. von Lindern M, et al. The translocation (6;9), associated with a specific subtype of acute myeloid leukemia, results in the fusion of two genes, dek and can, and the expression of a chimeric, leukemia-specific dek-can mRNA. Mol Cell Biol. 1992;12(4):1687-1697.

5. Waldmann T, Scholten I, Kappes F, Hu HG, Knippers R. The DEK protein - an abundant and ubiquitous constituent of mammalian chromatin. Gene. 2004;343(1):1-9.

6. Kappes F, et al. The DEK oncoprotein is a Su(var) that is essential to heterochromatin integrity. Genes Dev. 2011;25(7):673-678.

7. Mor-Vaknin N, et al. The DEK nuclear autoantigen is a secreted chemotactic factor. Mol Cell Biol. 2006;26(24):9484-9496.

8. Kappes F, et al. DEK is a poly(ADP-ribose) acceptor in apoptosis and mediates resistance to genotoxic stress. Mol Cell Biol. 2008;28(10):3245-3257.

9. Mor-Vaknin N, et al. DEK-targeting DNA aptamers as therapeutics for inflammatory arthritis. Nat Commun. 2017;8:14252.

10. Mor-Vaknin N, et al. High levels of DEK autoantibodies in sera of patients with polyarticular juvenile idiopathic arthritis and with early disease flares following cessation of anti-tumor necrosis factor therapy. Arthritis Rheumatol. 2018;70(4):594-605.

11. Bianchi ME, Crippa MP, Manfredi AA, Mezzapelle R, Rovere Querini P, Venereau E. Highmobility group box 1 protein orchestrates responses to tissue damage via inflammation, innate and adaptive immunity, and tissue repair. Immunol Rev. 2017;280(1):74-82. 